\title{
Market Design for Living-Donor Organ Exchanges: An Economic Policy Perspective
}

\author{
Tayfun Sönmez* $\quad \& \quad$ M. Utku Ünver ${ }^{\dagger}$ \\ June 2017 \\ for publication in Oxford Review of Economic Policy
}

\begin{abstract}
Within the last decade kidney exchanges emerged as a modality of transplantation to better utilize living donation possibilities as a cross disciplinary success of medical doctors and ethicists, market design economists, and computer scientists. This paper summarizes at which fronts these efforts have been successful and what needs to be done further to increase their impact. Also this paradigm is partially being applied to liver exchanges. There are other organs for which living donation is possible and gains from exchange can be much bigger than kidneys. Recent academic work on single-graft liver and dual-donor organ exchanges for lobar lung, dual-graft liver, and simultaneous liver-kidney transplantation are also discussed.
\end{abstract}

\section{Transplantation, Organ Donation, and Exchanges}

\subsection{Ethical Constraints and Donation}

In most of the world, buying and selling a body organ is illegal 11 Exchanges of human organs for valuable consideration have long been debated in philosophy, anthropology, theology, economics, and medicine. Since organ shortage is severe in most countries, for most economists a legal and regulated organ market would seem a natural solution. Most important objections against an organ market focus on ethical issues. For living human organs, projections predict the sellers to be

\footnotetext{
*Department of Economics, Boston College and Distinguished Research Fellow, Koç University; E-mail: sonmezt@bc.edu; URL: http://www.tayfunsonmez.net

${ }^{\dagger}$ Department of Economics, Boston College and Distinguished Research Fellow, Koç University; E-mail: unver@bc.edu; URL: http://www2.bc.edu/utku-unver

${ }^{1}$ For example, The National Organ Transplant Act (NOTA) of 1984 makes it an illegal activity in the US to exchange a body part for "valuable consideration." A notable exception is Iran, where a legal organ market is in place.
} 
poor and open to exploitation for monetary transactions; this is considered morally unacceptable. Moreover, living donation would most likely be crowded out in the sense that the sole providers of living human organs will be the sellers (for example, see Dougherty, 1987). On the other hand, Kerstein (2009)'s philosophical arguments focus on two Kantian principles. One principle forbids expressing disrespect for the dignity of humanity; the other forbids treating others merely as means. He also argues a person should not be allowed to engage in a voluntary transaction that will impair her future ability to engage in voluntary transactions. Such an example is one person selling himself into slavery for his family's needs. Kerstein argues that, even in a regulated market, the sale of an organ is such an economic transaction. ${ }^{2}$ Other objections focus on the demand side and the unethical nature of the right to buy (Scheper-Hughes, 2005). Paying for an organ is seen as a form of "buying life" by exploiting poor people, especially those from poor countries, and this is considered unethical. Market design emerged as a field that can be used to design institutions for welfare-improving transactions as a tool to mitigate the constraints imposed by such ethical and other constraints, besides many others (for example, see Li, 2017; Roth, 2007).

Although we summarized only the anti-market arguments, which seem to have prevailed until at least now, there are many pro-market views. Notably, Taylor (2005) proposes how a regulated market would work and why ethical counterarguments may not be as powerful as one would think. Indeed, some economists have argued for a market and even estimated the legal price of a kidney (for example, see Becker and Elías, 2007). Due to increased organ shortage, regulated market advocates and other economists have been coming up with innovative ideas for ethically acceptable welfare-improving schemes. Many nudges using well-accepted medical-incentive schemes, as well as the topic of this article, fall into this ethically acceptable category.

The no-sale constraint makes donation almost the only viable legal source of transplant organs. Many countries have developed sophisticated deceased-donor allocation schemes as a response. In opt-in consent countries (such as the US, Canada, the UK, Germany, the Netherlands, Israel, Taiwan, Japan, Venezuela), when healthy, a prospective deceased donor signals her willingness to donate by opting-in to a donor registry (which can only be overriden by her family upon her death and only in some systems). In opt-out consent countries, unless the individual overrides while she is healthy or her family overrides after her death, a suitable individual is automatically considered a deceased donor upon her death (such as Spain, France, Italy, Sweden, Greece, Poland, Russia, Singapore, Tunisia, Argentina, etc). In most countries, the deceased donor's suitable organs (usually brain dead due to an accident to the head that did not harm internal organs) are allocated through a centralized point system that prioritizes among waitlist patients.

Since the shortages are high and waiting times are long, many times a patient's loved ones come forward to directly donate to the patient. If she is deemed compatible with the patient, she can donate a piece or whole organ. Moreover, for some organs (such as a kidney or liver), living donation

\footnotetext{
${ }^{2}$ He cites Zargooshi (2001), who studies kidney sellers in Iran's legal market documenting that the sellers feel shame and have long-lasting psychological scars akin to prostitutes. It is also documented that black-market organ sellers in India made this decision in a dynamically inconsistent manner and ended up with worse health and economic situations (Goyal et al., 2002). Kerstein argues that poor regulation will also lead to problems akin to black markets.
} 
is a better alternative for the longevity of the transplanted organ. Living donation is the only viable option in many countries, where deceased-donation rates are very small. These countries include Eastern Asian countries (such as South Korea, Japan, China, and Hong Kong) and countries with Muslim populations (such as Turkey and Saudi Arabia). For these countries, living donation is de-facto the most important source of organs.

Living donation is practiced mostly for kidneys, livers, and also, to a lesser extent, for lungs. 3 Among the three, kidney transplantation requires the least intervention on the donor. For kidney donation, the living donor donates one of her two available kidneys. For both liver and lung, the donor's organ is cut and a piece of it (known as the graft) is transplanted to the patient. For liver donation, usually a lobe of the liver (which has a larger right lobe and a smaller left lobe) is taken. For lung donation, usually two living donors are required: each donor donates the lower lobe of one of her lungs and the two removed grafts are transplanted to the patient. There are other instances for which dual transplants are needed: if the size of the donor liver lobe is small, then two lobes can be taken from two living donors for a single successful transplant. Simultaneous liver-kidney transplants are practiced regularly from deceased donors (about 10\% of all deceased-donor liver transplants in the US) as such a procedure is proven to be better than sequential liver and kidney transplants for patients with dual-organ failure.

\subsection{Medical Constraints}

Despite all the progress in transplantation, in general, finding a medically compatible living donor is difficult. For example, the odds of a random pair being compatible for kidney transplantation are only about $50 \%$. This percentage goes down significantly for highly sensitized patients, who reject almost $99.9 \%$ of other tissue types and are known as high-PRA (panel reactive antibody) patients. Compatibility is governed by multiple and different mechanisms for different organs. The most common compatibility requirement is the blood-type (known as ABO in the medical community) compatibility, and it is required for all organs.

The common human blood types are determined by the existence or non-existence of two proteins known as A and B. Therefore, there are four blood types: O (referring to the lack of either antigen), A, B, and AB. A patient is blood-type compatible with a donor if he carries all of the donor's blood proteins. So an $\mathrm{O}$ donor can donate to all blood types, while an AB donor can donate only to AB. $\mathrm{A}$ and $\mathrm{B}$, on the other hand, can only donate to their own kind or to AB.

The second compatibility requirement is tissue-type compatibility. A person's tissue type is determined by human leukocyte antigens (HLA) in her DNA. If the patient does not carry pre-

\footnotetext{
${ }^{3}$ Bone marrow (and blood, as a matter of fact) is taken entirely from living donors for medical reasons. However, bone marrow donation involves a much smaller hardship and risk for the donor than the other organs discussed here. Therefore, unlike the organs discussed in this article, most bone marrow donors have no relationship with the patient and are true good Samaritans. Our article will focus on organs for which donation bears some significant cost (psychological, procedural, or related to health risk) so that most of the living donors are the family members or other loved ones of the patients.
} 


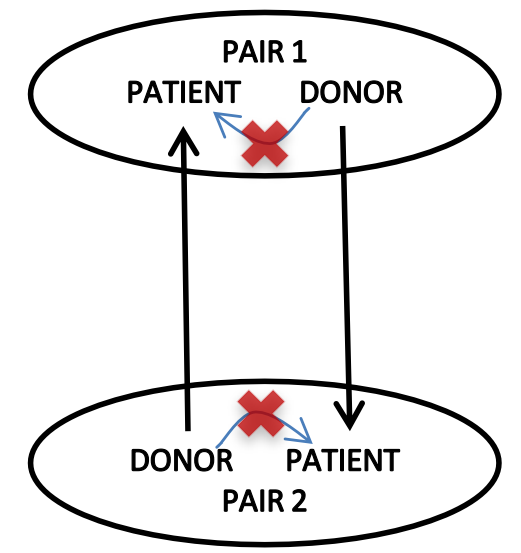

Figure 1: The depiction of a kidney exchange between two patients and their paired directed living donors who are incompatible with their own patients and compatible with each other's.

formed antibodies against the HLA of the donor in her blood, he accepts her organ (this is examined before the transplant through a blood test known as a crossmatch test; tissue-type compatibility is confirmed with a negative crossmatch result). For kidneys, this is an important compatibility concern. For lungs, there is no consensus about the this requirement's relevance. For liver, the crossmatch test is not important, and tissue compatibility does not play an important role.

The third compatibility requirement is size compatibility. Each donor's graft should be at the right size for the patient to receive it. For kidneys, this is not a big concern unless the patient or the donor have extreme body measurements. For liver and lungs, on the other hand, this is an important constraint. For these two organs, the donor(s)'s graft(s) should be large enough for the transplant to be successful.

Therefore, in many cases, willing donors cannot donate to their loved ones due to medical incompatibilities. Traditionally, such "directed" donors would not be utilized.

\subsection{Organ Exchanges and Outline}

In 1986, Rapaport (1986) proposed the formation of a database that would register the incompatible donors of kidney patients, so that incompatible patient-donor pairs could exchange donors to find a compatible match (see Figure 1).

With the exception of South Korea, this idea was not utilized until medical ethicists declared that so-called living-donor "kidney exchanges" do not violate no-sale laws, such as NOTA (see Ross and Woodle, 2000; Ross et al., 1997; The Authors for the Live Organ Donor Consensus Group, 2000).

The first kidney exchange was conducted in South Korea in 1991 (see Huh et al., 2008), followed by the establishment of a kidney-exchange program. The Netherlands also established a kidney exchange program early on (see De Klerk et al., 2005). While the first kidney exchange in the US 


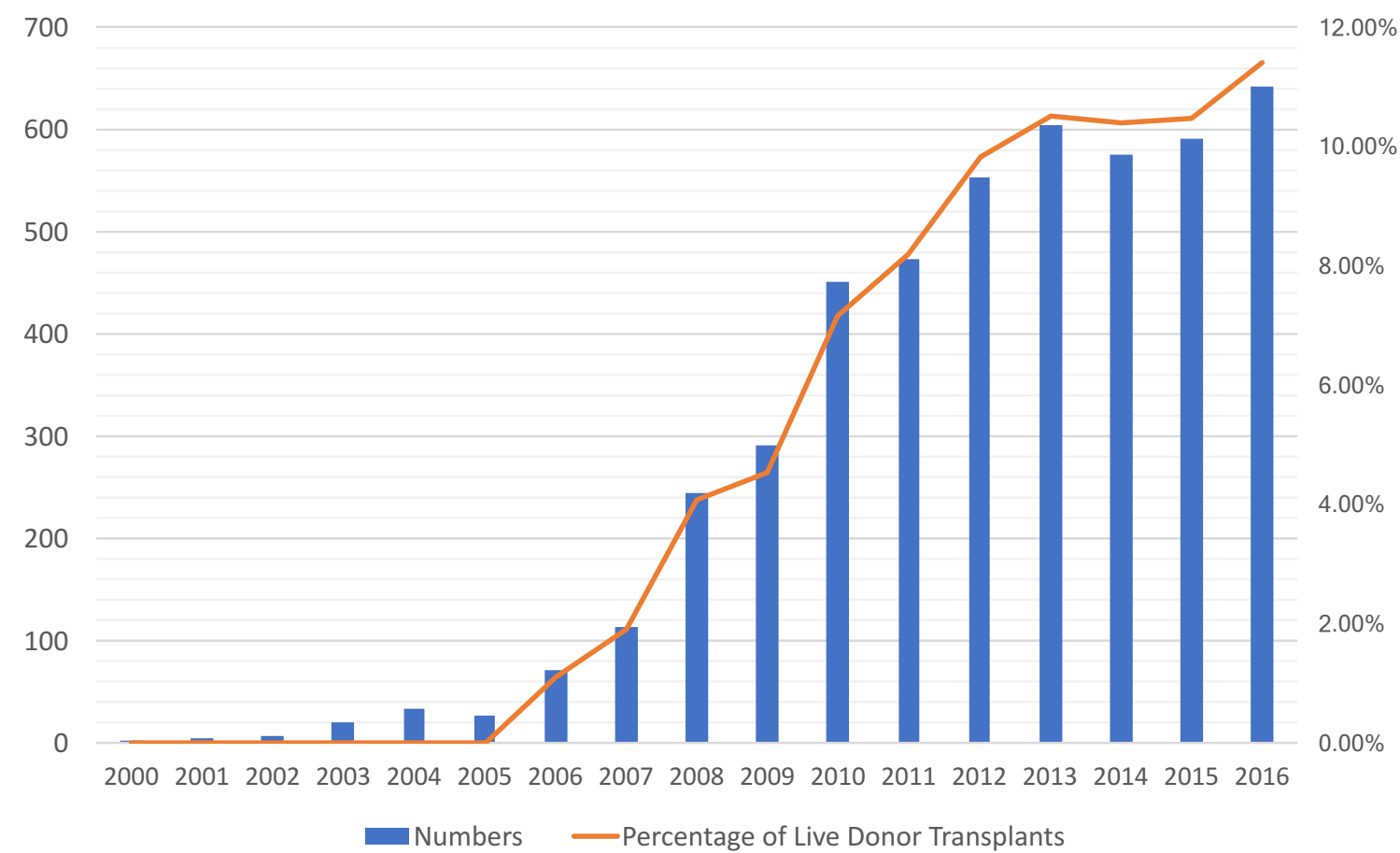

Figure 2: Number and percentage of transplants conducted through kidney exchanges in the USA, 20002016. OPTN- National Data retrieved from https://optn.transplant.hrsa.gov/data on 06-19-2017.

was carried out in 1994, the first formal program was established in Ohio in the 2000s. But until 2003, only a handful of transplants were conducted through exchanges in the US.

By 2016, this number was more than 640, about $11.4 \%$ of all living-donor kidney transplants (see Figure 2). This increase can be attributed to the adoption of market-design ideas in finding and conducting kidney exchanges in an organized manner. Market design emerged in the 1990s as a field of economics that uses formal economic theory, fueled by subfields such as game theory and mechanism design, to design methods to solve practical distribution and allocation problems, sometimes with the help of computer science and optimization methods.

This article will first illuminate in chronological order how the practice of kidney exchange was shaped by economics literature. Then it will discuss important problems that remain in kidney exchange and the recent developments in the literature that possibly address some of these problems.

The last parts of the article will review liver and lung exchange literature, along with multidonor organ exchange. Liver exchanges have occurred and formal programs exist in South Korea and Hong Kong. With the exception of one dual-graft liver exchange conducted in South Korea (Jung et al., 2014), multi-donor organ exchanges have not been practiced. These were proposed in a recent paper by Ergin, Sönmez, and Ünver (2017a). Exchanges' contribution in increasing the number of transplants can be substantial in such cases because directly recruiting multiple 
compatible donors is a bigger challenge..$^{4}$

\section{Kidney Exchange}

The progress of living-donor kidney exchange can be discussed under seven subtitles. The first four policy goals have been achieved to some degree while the last three have not been implemented to realize their full potential yet.

1. Policy achievements:

(a) Organization and optimization for kidney exchanges

(b) Utilizing gains from larger exchanges

(c) Integration of altruistic donors via exchange chains

(d) The role of kidney exchange when some medical incompatibilities are overcome by other means

2. Other important goals:

(a) Inclusion of compatible pairs for increased efficiency

(b) Higher efficiency via larger kidney-exchange programs

(c) Dynamic matching in kidney exchange

\subsection{Policy Achievements}

\subsubsection{Organization and Optimization for Kidney Exchanges}

Until 2003, the aggregate number of kidney exchanges conducted in the US were in the low teens. Ohio Solid Organ Consortium (OSOC), New England's transplant centers and the Johns Hopkins Transplant Center were some of the emerging leaders in conducting kidney exchanges. OSOC started the first organized program for kidney exchange. However, the role and importance of optimization was not initially clear in practice. A series of papers by Roth, Sönmez, and Ünver $(2004,2005 b$, 2007 ) within the context of mechanism design emphasized the importance of optimization.

From an economic point of view, a kidney exchange is one of the purest forms of trade, a barter concerning almost perfect substitute items from the point of view of an outsider, and, like any barter, it depends on the double coincidence of wants between two parties as famously noted by Jevons (1876) (see also Roth, Sönmez, and Ünver, 2007). The small number of conducted exchanges in this

\footnotetext{
${ }^{4}$ For more technical reviews of the market-design literature on kidney exchange alone, without the most recent developments, see Sönmez and Ünver 2013) and also Sönmez and Ünver (2011). For a computer science perspective, see Dickerson and Sandholm (2016). Also, this tutorial includes many references to computer science work.
} 


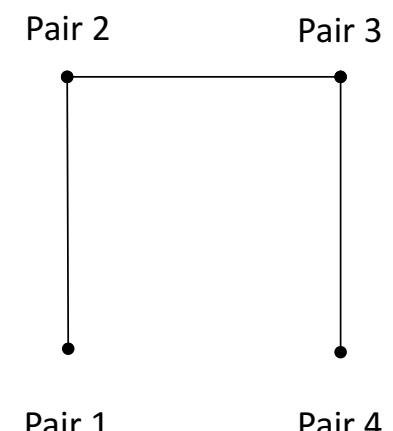

Figure 3: Consider an exchange pool with only 4 pairs. Let solid lines show the possible two-way exchanges. For example, Pair 1 - Pair 2 can exchange their donors while Pair 1 and Pair 4 cannot. In a decentralized market, if each exchange occurs first with equal probability, then with $1 / 3$ probability Pairs 2 and 3 will exchange, and match only 2 patients instead of the possible 4 (Pair 1 - Pair 2, Pair 3- Pair 2) along two separate exchanges.

period can be attributed to the fact that casually finding double (or more) coincidences of wants to create exchanges is difficult. Even if we take $p=50 \%$ as the rejection rate between a random donor and a patient, the chance of a feasible exchange between two random pairs is $(1-p)^{2}=25 \%$. As no large database existed, the discovery of exchange cycles was purely coincidental.

Two important and separate problems exist. One is the formation of an exchange pool of patient-donor pairs, akin to a market platform. OSOC, for example, had started to form such a pool early on in the US. New England and other places followed suit.

The other one is how efficient exchanges should be conducted in such a setting. There can be two approaches, a decentralized approach where pairs or their proxies, such as their doctors, chaotically try to arrange exchanges. In the absence of a medium of exchange and under certain time pressures when the pool is thin, efficiency of such a system is not clear at all. See Figure 3 for such an inefficiency in a thin, decentralized market.

Then why not try to centrally match the pairs by respecting medical, ethical, institutional, and economic criteria using market design? The rest of this paper focuses on this approach.

In this spirit, Roth, Sönmez, and Ünver (2004) introduced a new variant of the top-trading cycles, the mechanism of Abdulkadiroğlu and Sönmez (1999), whic was developed in the domain where dorm rooms are allocated to students on college campuses with and without initial property rights $5^{5}$ The latter mechanism was a generalization of both a serial dictatorship for allocation without initial property rights and David Gales's top trading cycles algorithm (mentioned in Shapley and Scarf, 1974) for allocation with initial property rights. The analogy between kidney exchange and dorm allocation is as follows: many rising sophomore, junior, and senior students already have dorm rooms but want to exchange them for better rooms if they could, and some students, such as new freshmen, do not have any, yet both types of students have collective rights over rooms vacated by

\footnotetext{
${ }^{5}$ See Cantillon (2017) (in the same issue) for a description of this algorithm.
} 


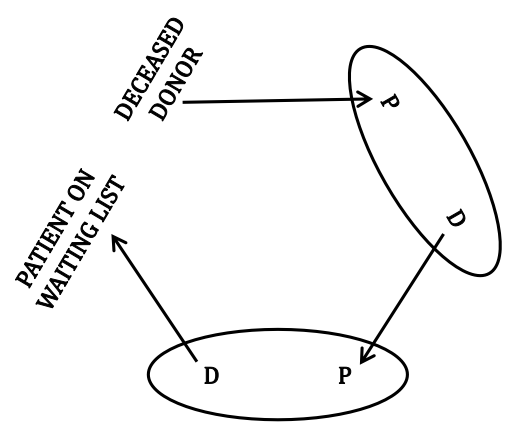

Figure 4: A list exchange chain involving two pairs

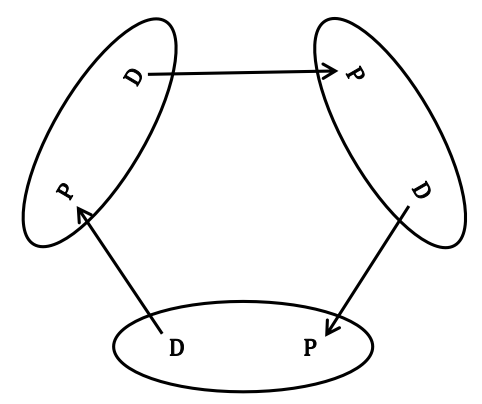

Figure 5: A three-way kidney exchange

graduating students. In kidney exchange and in kidney allocation in general, some patients have already-paired living donors but are incompatible or compatible but there could be other donors out there who can provide their transplants a longer survival term. Some other patients do not have paired living donors. There are deceased-donor or good-Samaritan-donor kidneys arriving over time, which are considered common endowment. Thus, integration of both deceased donation and kidney exchanges could result in chains, in which a deceased donor (or a good Samaritan) donates to a patient with a living donor, this living donor donates to another pair's patient, this patient's donor donates to another pair etc, and finally the last pair's donor donates to a patient waiting on the deceased-donor queue with the highest priority (see Figure 4). A simplified version of such a process with only one pair in the chain was already utilized by New England's transplant centers and known as list exchange. Of course, instead of a deceased donor, such a chain can start with a good Samaritan living donor, who is not paired with any patient. The second type of exchanges are cycles, in which a group of patient-donor pairs swap donors among themselves in a trade cycle (see Figure 5 for a three-way exchange). Roth, Sönmez, and Ünver (2004) demonstrated high gains from organized exchanges, where chains and cycles were possible, instead of myopic random organization of two-way exchanges (i.e., only two pairs swapping donors) and short list exchanges.

The only problem with this approach is that it could lead to long cycles and chains. An organ donation is a gift, and a donor can always change her mind. Therefore, it is not contractable. If all transplants in a cycle are not conducted simultaneously, a donor whose patient already received 
a kidney can back out of an already-planned transplant, harming at least one patient whose donor already donated; she loses a living donor but cannot receive a living-donor transplant. To prevent this, all transplants in a cycle are conducted simultaneously. Hence, because of logistical difficulties in organizing multiple transplant teams simultaneously, it is infeasible to realize large exchange cycles.

To remedy this problem, Roth, Sönmez, and Ünver (2005b) proposed using methods from combinatorial optimization, such as cardinality matching algorithm of Edmonds (1965), to conduct only two-way exchanges. Moreover, based on Gallai (1963, 1964)-Edmonds (1965) decomposition of graphs, other objectives can also be achieved besides maximizing the number of transplants. As a result a version of this approach, known as priority mechanism, was utilized in the newly established New England Program for Kidney Exchange (NEPKE) beginning in 2004, the first exchange program using optimization.

We introduce some notation that will be useful to keep things concrete. Let $P$ be the set of patient-donor pairs that would like to participate in exchange. Suppose the pairs are ordered according to some real-valued priority function $\pi$ with no ties. We say pair $i$ has higher priority than pair $j$ if $\pi(i)>\pi(j)$. All feasible two-way exchanges among pairs can be represented as a non-directed graph $E$, as in Figure 3. We say a two-way exchange $(i, j) \in E$ if pairs $i$ and $j$ can participate in a feasible two-way exchange. This model assumes a pair is indifferent between two feasible exchanges in which it can participate. A matching $\mu \subseteq E$ is a collection of two-way exchanges such that a pair can be part of at most one exchange. By abuse of terminology, we say $i \in \mu$ if $i$ is matched in an exchange of $\mu$. We find a matching that maximizes the number of patients matched, starting with an empty set of agents $I^{0}=\emptyset$ that we update in each step and that we call the set of simultaneously matchable patients.

We introduce the priority two-way exchange mechanism of Roth, Sönmez, and Ünver (2005b) as follows:

Step 1. If there is an exchange including the highest priority pair $i$ in $E$ then $I^{1}=\{i\}$; otherwise $I^{1}=\emptyset$.

Step k. If there is a matching that matches all pairs in $I^{k-1}$ as well as k'th highest priority pair $j$ (and maybe other pairs as well) then $I^{k}=I^{k-1} \cup\{j\}$; otherwise $I^{k}=I^{k-1}$.

When the mechanism concludes in Step $|P|$, we have determined a subset of pairs $I^{|P|} \subseteq P$ that are simultaneously matchable. Any matching of these pairs is called a priority matching. The main advantage of this mechanism is that it maximizes the total weight of priorities of pairs matched $\sum_{i \in \mu} \pi(i)$, as well as the number of transplants. Thus, even if the sum of priorities has a cardinal meaning, such as a welfare measure, without loss of generality we can totally ignore it.

If some patients bring more than one donor to the exchange pool, the mechanism can be extended. For each patient, it is a dominant strategy to bring all of his paired donors under this 
mechanism. If the patient's doctors are determining his options for exchange, it is also to the patient's best advantage to be truthful about them.

Determination of whether a new patient is matchable in each step, in addition to the previously committed agents, can be determined through a computational process called augmentation of alternating exchanges. The Edmonds (1965) algorithm that determines an arbitrary matching that maximizes the number of transplants works using a variant of this method.

Question and solution of this computational problem can be posed as follows: Suppose in Step k-1, we already determined a matching $\mu^{k-1}$ that matches all pairs in $I^{k-1}$ (and maybe more).

Suppose $j$ is k'th highest-priority pair. if $j$ is already matched in $\mu^{k-1}$, we are done. Suppose not. Is there a path of exchanges in and out of $\mu^{k-1}$ involving different pairs $j=i^{0}, i^{1}, \ldots, i^{l}, j^{\prime}$ where $l \geq 0$ (so comes the name alternating), such that

$$
\begin{aligned}
& \left(j, i^{1}\right) \in E \backslash \mu^{k-1} \\
& \left(i^{1}, i^{2}\right) \in \mu^{k-1} \\
& \left(i^{2}, i^{3}\right) \in E \backslash \mu^{k-1} \\
& \quad \vdots \\
& \left(i^{l-1}, i^{l}\right) \in \mu^{k-1} \\
& \left(i^{l}, j^{\prime}\right) \in E \backslash \mu^{k-1} \text { and } j^{\prime} \text { not matched by } \mu^{k-1}, \quad \text { or } \quad i^{l} \notin I^{k-1} ?
\end{aligned}
$$

It turns out such a path of exchanges exists if and only if we can match $j$ in addition to all agents in $I^{k-1}$. We choose exchanges above that are not in $\mu^{k-1}$ instead of the ones in it and do not change the rest of the exchanges in $\mu^{k-1}$. Let the resulting matching be $\mu^{k}$. This inclusion exclusion is the augmentation. It can be done in polynomial time (Edmonds, 1965).

In addition to NEPKE, other transplant centers also accepted this approach. For example, the Johns Hopkins University Transplant Center adopted a similar approach (see Segev et al., 2005). The difference of the Johns Hopkins approach and Roth, Sönmez, and Ünver's was that instead of priority matching for pairs to maximize the two-way exchange number, they proposed edgeweighted matching, using another application of Edmond's algorithm. Each exchange was weighted by a number, and then a matching that maximized the sum of edge weights was found. A recent paper by Okumura (2014) showed that priority matching can also be found directly using Edmond's edge-weighted method, which we will not discuss in this article.

\subsubsection{Utilizing Gains from Larger Exchanges}

While it will be logistically difficult to conduct very large exchanges, three-way exchanges can also be feasibly conducted in many US hospitals. Moreover, economic intuition suggests that arbitrarily limiting the size of exchanges may lead to significant efficiency losses.

Indeed, the NEPKE algorithm did not only find two-way priority matchings. The algorithm 
was tweaked to find two\&three-way priority matchings. Suppose a three-way exchange is denoted by a triple of pairs $(h, i, j)$ where $h$ donates to $i, i$ donates to $j$, and $j$ donates to $h$. Using the previous subsection's notation, let $E$ denote the set of feasible two\&three-way exchanges. Then the definition of the priority mechanism extends to priority two\&three-way exchange mechanism used in NEPKE.

However, it is not easy to execute each step in the definition of the mechanism individually, unlike the two-way version. Therefore, the whole problem can be solved in one step using integer programming techniques that we will discuss later.

Roth, Sönmez, and Ünver 2007) formulated the rationale for this approach more formally. If the underlying patient population in an exchange pool follows the distribution of new entrants, then almost all gains from exchange can be exhausted using only two\&three-way exchanges. The theoretical model showed that when there are $\mathrm{n}$ blood types, any maximal matching can be reconstructed to match the same pairs and have no larger than n-way exchanges. Since there are 4 blood types and AB blood type is very rare in the US (about only $4 \%$ of the population), two\&three-way exchanges can utilize almost all gains from exchange (see Table 1, reported in Section 2.2, to see approximate gains of utilization of different size constraints on kidney exchange).

Saidman et al. (2006) showed in more realistic simulations and exchange pools that conclusions of this theoretical analysis were fairly accurate.

As a result, not only NEPKE, but the newly consolidated Alliance for Paired Kidney Donation (APKD) (a successor of OSOC) also adopted the methods proposed by Roth, Sönmez, and Ünver (2007). Johns Hopkins and the newly established National Exchange Program followed suit. National Exchange Program, which aims to have a large pool, uses Abraham, Blum, and Sandholm (2007)'s interpretation (see below).

As we mentioned, such an optimization problem can only be solved using integer programming algorithms unlike the case of two-way exchanges (for which polynomial algorithms, such as Edmonds', exist) (see Roth, Sönmez, and Ünver, 2007). Let $E^{k}$ be the set of feasible exchanges of size less than or equal to any given fixed length $k \leq|N|$. Let $\mathcal{M}\left(E^{k}\right)$ be the set of feasible matchings. We depict such an integer program as follows:

$$
\max _{\mu \in \mathcal{M}\left(E^{k}\right)} \sum_{e \in \mu} w(e) \cdot \mathbf{1}\{e \in \mu\}
$$

Here $\mathbb{1}\{\mathcal{X}\}$ is the indicator function, taking value 1 if $\mathcal{X}$ is a true statement, and otherwise taking value 0 ; while $w(e)$ is a real number weight associated with exchange cycle $e$.

Above, the weights can be chosen according to policy objectives. For example, if we set $w(e)$ to the number of transplants in exchange $e$ (i.e, for a two-way exchange $e$, we set $w(e)=2$ ), the integer program finds a matching that maximizes the number of transplants. We can also set the weights lexicographically based on the priorities of agents: Suppose we set $w(e)=\sum_{i \in e} \pi(i)$. If all exchanges are of size 2 , then the solution is a priority matching. If $k>2$, then not all priority 
functions can be used as $\pi$ in the above formulation to find a priority matching. Once $\pi$ is calibrated accordingly, we can find one. This was used in NEPKE starting in 2005.

Suppose $m$ is the number of transplants in the transplant-maximizing matching found as a solution of the above problem when each weight is the size of the exchange. There can be many maximum matchings. Suppose our goal is to find the maximum matching that maximizes the transplant center's welfare criterion. Let $\left\{w^{\prime}(e)\right\}_{e \in E^{k}}$ be such weights reflecting the real welfare criteria used by the system. Then, we can solve a second optimization problem using these weights with a constraint

$$
|\{i \in \mu\}|=m \text {. }
$$

This is the approach utilized in APKD starting from 2005 until now (for example, see Anderson et al., 2015b).

As we will see, set $E^{k}$ can be defined to include other types of exchanges that we introduce below.

A challenge with such an integer-programming approach is that computationally finding maximal matchings with maximum exchange sizes greater than 2 is NP-complete. This result was proven and a scalable algorithm was proposed to solve the optimization problem in large pools by Abraham, Blum, and Sandholm (2007).

The UK is another country with an extensive kidney-exchange program. They use the ideas explained in subsections 2.1.1 to 2.1.3 to utilize both short altruistic-donor chains and three\&fourway exchanges. The optimization objectives are slightly different in the UK. They care about obtaining small cycles, as false-compatibilities are possible in data (i.e., those transplants that do not get carried out eventually because of a misidentified tissue-type compatibility). If one transplant falls apart, then the whole cycle fails to be realized. Having smaller cycles is a safeguard against such data errors; however, they come with the caveat of limiting the number of transplants. Manlove and O'Malley (2012) detail how the UK system works and explain the safeguards they built in. Chosen four-way cycles have three-way cycles embedded in them so that if one of the transplants fails due to false-compatibility then the rest can be carried out as a three-way cycle. They report simulations on real data sets showing that four-way exchanges would result in a significant increase in the number of patients receiving transplants.

\subsubsection{Integration of Altruistic Donors via Exchange Chains}

In the US, there is a considerable number of (good Samaritan) altruistic donors who wish to donate their kidneys to patients they do not know. In the past, these kidneys were utilized through the waiting list similar to deceased-donor kidneys.

However, as in the case of list-exchange chains, one can think of creating altruistic-donor chains to help more than one patient (see Figure 6). Such chains were indeed conducted by Johns Hopkins in May 2005 (Montgomery et al., 2006), by New York Presbyterian hospital in May 2006 (press 


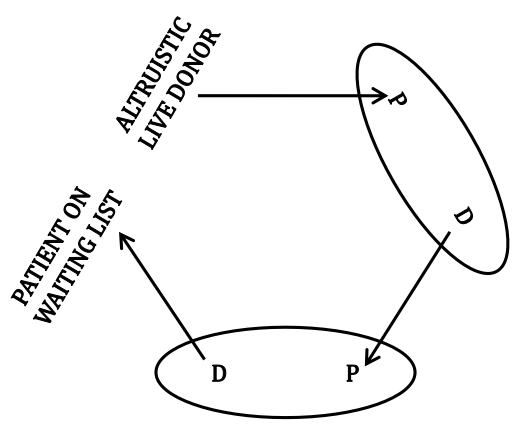

Figure 6: A 'closed' altruistic-donor chain involving two pairs

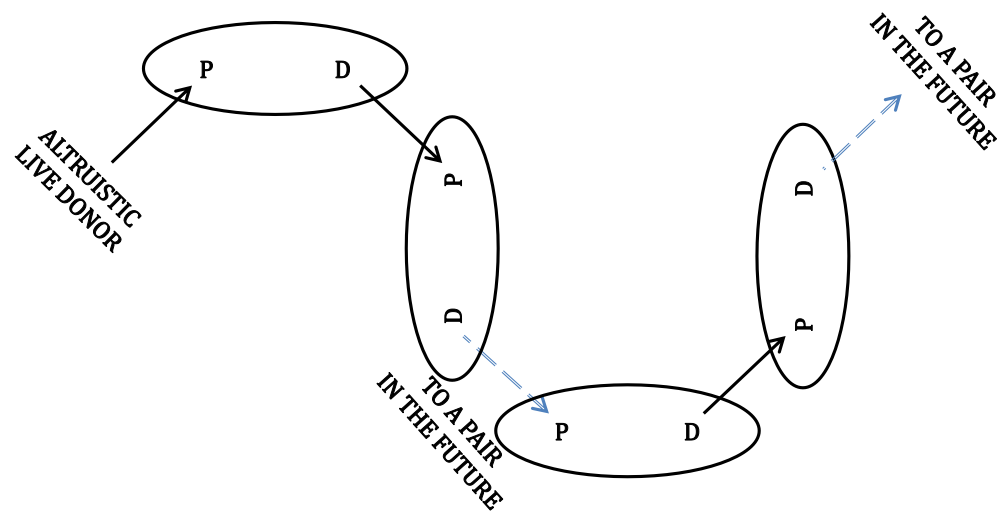

Figure 7: An 'open' non-simultaneous altruistic-donor chain

release), and by NEPKE in July 2006.

Different from exchange cycles in which a number of pairs trades kidneys among each other, there is no real simultaneous-transplant necessity in a list-exchange or altruistic-donor chain. The transplants can be done consecutively, starting from the altruistic donor's donation, continuing in the direction of the arrows in Figure 6. Hence, no patient gives up her donor before receiving a transplant. Even if one donor in the chain backs out, at least one patient will benefit from the altruistic/deceased donor. The length of the chain can be set as arbitrarily long as one wishes. This was initially proposed by Roth et al. (2006).

Moreover, the last donor in the chain does not need to donate back to the waiting list. He can serve as a 'bridge' donor for any future donation, almost like the initial altruistic donor (see Figure 7). Following Roth et al. (2006), non-simultaneous altruistic-donor chains started in the APKD in 2007. As the first of them, a 10-pair chain was formed (see Rees et al., 2009) $6-6$

Although Roth, Sönmez, and Ünver (2007) theoretically showed three-way exchanges are almost sufficient to obtain all gains of exchange, this theoretical result relies on two important assumptions.

${ }^{6}$ See Sack $(2012)$ for a more recent 30-pair chain's story.

${ }^{7}$ Also see Melcher et al. (2016) for a more recent push to make non-simultaneous deceased-donor chains a reality. 
The first one is that the pool is sufficiently large, and the other is that the pool evolves under a long-run assumption in which new pairs arrive over time while some of the existing ones are periodically matched, using optimal-exchange mechanisms. However, either assumption may fail in practice. The first assumption may fail in a fragmented exchange marketplace where multiple exchange clearinghouses function, as in the US. The second assumption may fail when the pool is not mature at the start of a market. Many patients have blood types that are difficult to match (such as O) annd they have less-desired donors (such as blood type A). Some patients have many preformed antibodies that reject the tissue types of almost all random donors. Thus, a small pool is detrimental for finding matches for such patients. Especially when the first assumption fails and the second assumption is satisfied, such 'difficult-to-match' pairs accumulate disproportionately in the pool. Indeed, if in general the accumulation rate of such pairs (as compared to the rate at which the pool gets bigger) is disproportionately large, the benefits from larger exchanges may be substantial. Indeed, the innovation of non-simultaneous deceased/altruistic living-donor chains may lead to large chains that can non-trivially increase the number of transplants. A model rationalizing how utilizing larger exchanges/chains can help save more patients was introduced in Ashlagi et al. (2012). They proposed a population-formation heuristic, which is borrowed from sparse but large random graph formation models in graph theory, to explain how such larger exchanges can be beneficial in such pools.

\subsubsection{The Role of Kidney Exchange When Medical Incompatibilities are Overcome by Other Means}

It turns out that A blood type consists of many different subtypes. In particular, A1 is the largest subtype, covering $80 \%$ of the A population. We will refer to the other subtypes simply as A2, as this subtype makes up $19 \%$ of all A, and the remaining $1 \%$ are similar to subtype A2 in terms of their immunological properties. The interesting feature of A2 is that a subtype-A2 donor can donate to $\mathrm{B}$ and $\mathrm{O}$ blood-type patients if the antibody level, known as titer value, of such patients against A blood protein is not too high. The patient has to have a consistently low titer value for at least 6 months to be eligible for such a transplant. Because B type patients wait longest in the deceased-donor waiting list in the US, the newly adopted kidney allocation policy prioritizes subtype-A2 deceased-donor kidneys for blood-type B patients (but not for blood-type O patients). Because of this, the titer history is readily available for B patients but not for O. Thus, this policy could be unintentionally extended to kidney exchange. That is because blood-type $\mathrm{O}$ patients (unlike blood-type B patients) will lack the tests that are necessary to receive subtype-A2 kidneys. A recent paper by Sönmez, Ünver, and Yllmaz (2016) shows that as long as the number of A patient - B donor pairs is larger than the number of B patient - A donor pairs, such a policy could decrease the total number of transplants in a population using living donation directly and through exchange. The rationale is as follows: Since A2's can directly donate to B's, the B-A2 pairs that would otherwise enter the exchange pool now become compatible. In the counterfactual, such a pair would most likely be matched with an A-B pair in a two-way exchange, as B-A's are in short supply. 
Now instead of additionally saving one pair, the B-A2 pair only saves itself. However, if O patients were also eligible for A2 kidneys with extensive titer value histories, then this would be better than allowing only A2 to B donations. Since there are typically many O patient - A donor pairs in the pools, this would not adversely affect exchange. The best policy suggestion given by this paper is that if the cost of testing for A subtyping of the living donor, which is an expensive procedure as it requires molecular-level tests unlike simple blood-type tests, was paid by an insurance company after a pair with an A donor and B or O patient decides to try its chances first for an exchange, then the number of transplants would increase considerably under the A2 to B or O donation policies.

Another hot topic in the discussion of kidney-exchange implementation is the patient's use of medications before the transplant to get rid of antibodies that cause incompatibilities. Some countries, led by South Korea and Japan, use expensive treatments to filter all types of antibodies from the blood of the patient, so that medical incompatibility is no longer a problem. However, this cleansing procedure is very expensive, on the order of at least half of the transplant cost itself. Moreover, medical literature is not in consensus that the longevity of a kidney graft after an incompatible transplant is as long as it is after a compatible one. Recently, Chun, Heo, and

Hong (2016) find mechanisms that would use a limited number of such filtering operations while maximizing the number of transplants under different kidney-exchange procedures.

Andersson and Kratz 2016) studies a similar problem. In Sweden, Australia, and some other countries, a compatibility standard that allows blood-type incompatibility in transplantation is utilized, while tissue-type incompatibility remains a huge barrier. Extensive titer-value testing is required for patients in such a case and filtering the blood of the anti-A and anti-B blood protein antibodies. Supposing patients prefer compatible transplants to blood-type incompatible transplants, they show that a two-way priority matching that is Pareto-efficient and maximizes the number of compatible transplants can be found through the weighted matching algorithm of Edmonds (1965). Indeed, among Scandinavian countries, an exchange program was established recently utilizing this approach, with the help of the authors of this paper.

These studies show that there is a room for utilization of kidney exchange even if medical incompatibilities cease to be hard constraints. Due to soft constraints, such as the high costs of filtering procedures or preference toward compatible transplants, the role of kidney exchange does not diminish much.

\subsection{Other Important Goals}

Nowadays about half of the transplants utilizing kidney exchanges are done through altruistic-donor chains, while the rest are done through two-way and three-way kidney exchanges. This total number is more than 600 annually and more than $10 \%$ of all living-donor transplants in the US.

Although kidney exchange became a successful transplantation modality with market design playing an important role, still the full potential of exchanges has not been fulfilled. In this subsec- 
tion, in the order of importance, we survey what can be done to fulfill its potential.

Although, we write these as future goals, for many of them significant strides have been taken to make them a reality. As of this writing, they are not implemented in their full power, so we will denote them as future goals.

\subsubsection{Inclusion of Compatible Pairs for Increased Efficiency}

First and foremost, if a patient has a compatible donor, she generally does not participate in the exchange pool. Even though she can find a better matched (in terms of HLA matching) or a younger donor through kidney exchange, this option has not been utilized much except in a few places, such as the Texas Transplant Institute in San Antonio, TX (see Steinberg, 2011).

After Ross and Woodle (2000) proposed the idea of incorporating compatible pairs into exchange (which they called 'altruistically unbalanced exchange'), Roth, Sönmez, and Ünver (2004) introduced the TTCC mechanism that includes compatible pairs in an exchange only if the patient of the pair receives a donor graft better than the graft of her paired donor in terms of long-term survival. Roth, Sönmez, and Ünver (2005a) demonstrated that the benefits of having compatible pairs in exchange can be substantially large. Indeed, there is an inherent asymmetry in the formation of exchange pools, in the absence of compatible pairs. A pair with an A blood-type donor and an $\mathrm{O}$ blood-type patient always enters an exchange pool as the $\mathrm{O}$ patient cannot receive from the A donor due to blood-type incompatibility. On the other hand, a pair with an O blood-type donor and and A blood-type patient enters the exchange pool only if there is a tissue-type incompatibility between the patient and the donor, which happens with a probability on the order of 10\%. Thus, if the patient-donor pair blood types were uncorrelated and if the probability of being a donor as well as the probability of being a patient were identical across all blood types, one would expect a 10-to-1 ratio between O-A patient-donor pairs and A-O patient-donor pairs. The empirical observations qualitatively support these findings. If almost all compatible pairs participate in exchange, we can save most of the pairs. Table 1 shows some summary statistics based on simulation results for comparative marginal gains from kidney exchange when compatible pairs are absent vs. kidney exchange when compatible pairs participate (Roth, Sönmez, and Ünver, 2005a). We present in Table 1 potential gains from various exchange modalities approximated from simulations reported in Roth, Sönmez, and Ünver 2005a, 2007). Out of 100 pairs randomly formed using the US population-generating distributions, about 47.5 pairs are compatible. When such pairs do not enter the exchange pool, two-way exchanges alone match an additional 26 patients on average, while the use of larger exchanges helps to match 31.5-32 pairs. When the initial direct donations involving compatible pairs are also accounted for, the totals are 73.5 and 79-79.5 pairs matched under two-way exchanges and exchanges with larger exchange sizes, respectively. On the other hand, if compatible pairs first participate in exchange and then donors of remaining unmatched compatible

pairs directly donate to their patients, these two numbers are 91.5 and 94, respectively. Thus, the potential inclusion of compatible pairs in exchange would be the most important innovation since 


\begin{tabular}{|c|c|c|c|}
\hline Regime & $\begin{array}{c}\text { Transplant } \\
\text { Contribution }\end{array}$ & $\begin{array}{c}\text { Marginal } \\
\text { Transplants }\end{array}$ & $\begin{array}{c}\text { Total } \\
\text { Transplants }\end{array}$ \\
\hline \multicolumn{4}{|c|}{ First direct donation, then exchange with incompatible pairs } \\
\hline Direct donation & 47.5 & +47.5 & 47.5 \\
\hline then two-way exchange & 26 & +26 & 73.5 \\
\hline or then two\&three-way exchange & 31.5 & +5.5 & 79 \\
\hline or then two\&three\&four-way exchange & 32 & +0.5 & 79.5 \\
\hline or then unrestricted exchange & 32 & +0 & 79.5 \\
\hline \multicolumn{4}{|c|}{ First exchange with all pairs, then remaining direct donation } \\
\hline $\begin{array}{l}\text { Two-way exchange } \\
\text { then direct donation }\end{array}$ & 91.5 & +12 & 91.5 \\
\hline $\begin{array}{l}\text { Unrestricted exchange, } \\
\text { then direct donation }\end{array}$ & 94 & +2.5 & 94 \\
\hline
\end{tabular}

Table 1: Approximate marginal gains from different cycle sizes for regular kidney exchange and kidney exchange when compatible pairs participate out of 100 randomly generated pairs using the US population statistics. Standard deviations are not available, as these are approximated using a meta-analysis.

the introduction of exchange itself.

Different aspects of inclusion of compatible pairs in exchange have been inspected in the literature. Gentry et al. (2007) run a US-size calibrated simulation to assess how many additional transplants would be feasible if compatible pairs participated in exchange. Sönmez and Ünver (2014) inspect the structure of efficient two-way exchange matchings when compatible pairs are included and propose a priority mechanism. Sönmez, Ünver, and Yenmez (2017a) propose an incentivization scheme to include compatible pairs into exchange. Patients with compatible donors need to wait in an exchange pool if they decide to participate in exchange. Moreover, they are possibly risk averse to receive some other donor's kidney while their donor's kidney is already available. These factors contribute their unwillingness to participate in exchange. The authors propose to incentivize their participation through an acceptable insurance tool for living donation. Regular living donors already have already insurance against a possible organ failure in the future. If their only available kidney fails after they donate the other one, they receive priority on the waiting list. Similarly, the same kind of insurance can be given to the patient of a compatible pair: if the kidney she receives from a different donor fails in the future for any reason, she can be given priority on the waiting list for a deceased-donor organ.

A similar version of such a time trade was recently realized in Los Angeles (press release): A young person with a kidney disease, but not in need of a kidney right now, had an old paired donor. If she needs a kidney in the future, this paired donor will potentially either be dead or unsuitable for donation at that time. Thus, she traded her donor's kidney now for an insurance to get priority in the future, if and when she needs a kidney transplant.

Sönmez and Ünver 2014) extend the priority mechanism and theoretical results of Roth, Sönmez, and Ünver (2005b) to the esetting when compatible pairs participate in exchange in a 
two-way matching setting when all compatible donors are indifferent but patients have an inherent bias toward their own compatible donor. Nicolò and Rodriguez-Álvarez (2017) adopt age-based preferences (i.e., all patients prefer younger donors to older ones) in the design of priority two-exchange mechanisms to accommodate restricted cycle sizes for incentivizing patients with compatible donors to participate by exchanging their donors for younger ones.

The main difficulty to overcome including compatible pairs is to persuade them of the marginal benefit of exchange. As finding and arranging exchanges can be time consuming, this is a deterrent for compatible pairs to participate in exchange. Several successful proof of concepts have been reported in the medical literature, signaling possible future extension of inclusion of compatible pairs, who signaled their willingness to participate in surveys (for example, see Ratner et al., 2010). Moreover, many medical papers also endorsed altruistically unbalanced exchanges (for example, see Veatch, 2006, Kranenburg et al., 2006, Steinberg, 2011, and Ferrari et al., 2017) and discussed their potential benefits.

\subsubsection{Higher Efficiency via Larger Kidney-Exchange Programs}

The federal National Exchange Program in the US was established in 2010, while other independent programs were established beginning in 2003. The main reason for this delay is that National Organ Transplant Act (NOTA) of 1984 did not clarify whether a living-donor organ exchange violated the law against the exchange of human organs for valuable consideration. While most ethicists (for example, see Ross et al., 1997 and Ross and Woodle, 2000) thought that they did not, an amendment to NOTA was not introduced until 2007. Thus, the establishment of the National Program was delayed.

The National Program is currently operated by United Network for Organ Sharing (UNOS), the same federal contractor that oversees deceased-donor allocation in the US. NEPKE dissolved itself in the national program to become the de facto National Program, while other regional exchange programs continue to operate. Most notably, an independent program, the National Kidney Registry, which is centered in New York, rose to prominence along with APKD. Frequently, a transplant center participates in multiple programs at the same time, as well as internally matching its pairs through its own paired-exchange program.

The upside of this fragmentation is that smaller, independent programs can experiment novel exchange paradigms without manny bureaucratic hurdles. Indeed, most novelties were adopted after some experience in such programs.

The main downside of this fragmentation is preventing the creation of a single large pool. The consensus view among researchers is that the larger the pool, the better the achievable gains from exchange. Especially if the numbers of difficult-to-match patients grow disproportionately over time, larger pool sizes will better exploit the full advantages of using exchange.

Besides the time and path dependence of the development of kidney exchange in the US, and 


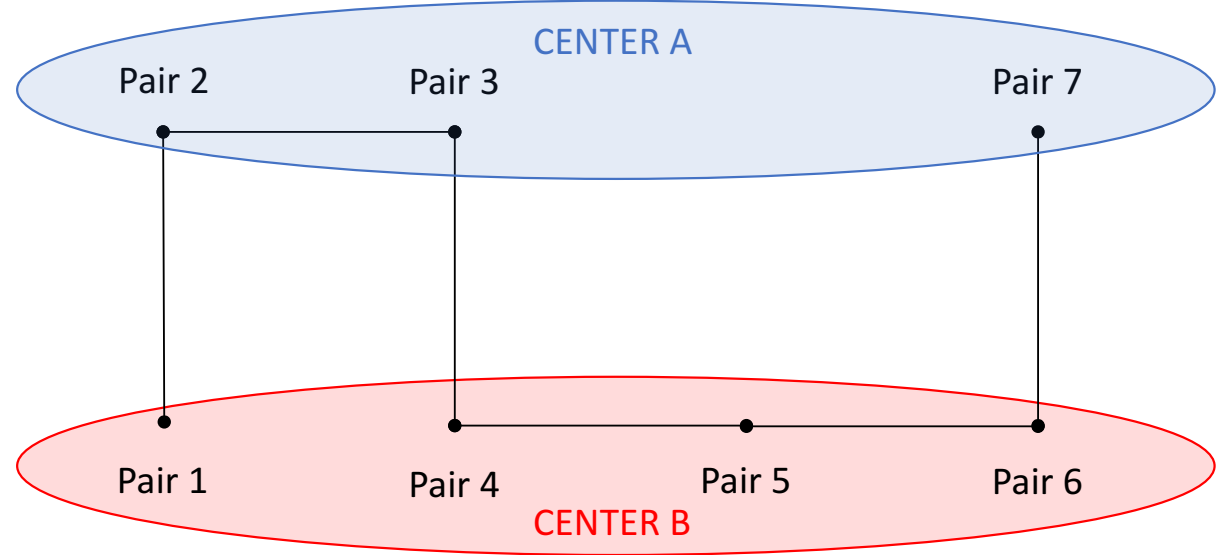

Figure 8: Suppose there are 7 pairs with the possible exchanges between the pairs denoted as in the figure (only two-way exchanges are feasible). Suppose Transplant Center A has 3 patient-donor pairs: Pairs 2, 3, and 7; while Transplant Center B has 4 patient-donor pairs: Pairs 1, 4, 5, and 6. Each center wishes to maximize the number of transplants for its patients (i.e., the ones it conducts). They can report whichever pair they like to the centralized system while they conduct exchanges among the pairs they did not report internally, and the centralized system chooses a transplant-maximizing matching among the reported pairs. We seek a Nash equilibrium of a reporting game. If the centers report all their pairs, 6 total pairs can be matched, either (a) 2 from Center A and 4 from Center B (i.e., outcome matching is $\{(1,2),(3,4),(5,6)\})$, or (b) 3 from Center A and 3 from Center B (i.e., outcome matching is $\{(2,3),(4,5),(6,7)\})$. If the first matching is chosen, then Center A could withhold Pairs 2 and 3, which it internally matches; then it guarantees Pair 7 is matched as the centralized system now has to choose $\{(4,5),(6,7)\}$. So Center A benefits by having one more of its pairs matched by withholding information (at the cost of Pair 1 from Center B remaining unmatched). If the second matching is chosen by the centralized system, then Center B could withhold Pairs 5 and 6 and match them internally. Then the centralized system will have to choose matching $\{(1,2),(3,4)\}$. Center B benefits by having all of its pairs matched (at the cost of Pair 7 from Center A being unmatched). So truthfully reporting all pairs to the centralized system is not a dominant strategy under any transplant-maximizing centralized system.

the bureaucratic advantages of having a fragmented market of independent exchange programs, one important hurdle in front of creating a unified exchange program could be a theoretical insight. Roth, Sönmez, and Ünver (2005c) show that in general there is no exchange mechanism that maximizes the number of transplants and makes full participation a dominant strategy for transplant centers (see Figure 8). This result hinges on 1) the ability of transplant centers to conduct exchanges internally in their own pool of patients, and 2) transplant centers only caring about maximizing the number of their own patients receiving transplants. Indeed, the problem may turn into a 'lemons market' adverse-selection problem in which only difficult-to-match pairs are sent to the centralized program, while easy-to-match pairs are matched internally by the transplant centers. The current composition of the national program exchange pool vs that of independent program pools gives some empirical evidence of the validity of this theoretical insight.

To overcome this difficulty, several different ideas are proposed. Ashlagi and Roth (2014) propose a centralized exchange mechanism that achieves Bayesian incentive compatibility in an approximate sense for the above participation problem. Caragiannis, Filos-Ratsikas, and Procaccia $(2015)$ and 
Ashlagi et al. (2015) propose strategy-proof lottery mechanisms to achieve a higher level of efficiency (greater or equal to $2 / 3$ of full efficiency) than deterministic strategy-proof mechanisms, which have the worst case of matching half of the patients that are matched under a maximal mechanism. Toulis and Parkes (2015) show, using random graph methods, that the number of patients that can benefit from two-way exchange scales from pooling as the square-root of the number of pairs in each center. They also propose a centralized two\&three-way exchange mechanism that would make it a dominant strategy for large centers to participate truthfully while maximizing the number of transplants.

Another interesting idea is making use of the incentivization of compatible pairs idea proposed in Sönmez, Ünver, and Yenmez 2017a mentioned in the previous subsection to create a large single-exchange clearinghouse. Recall that the idea was that patients of compatible pairs would be insured against failure of the transplant by being sent to the top of the deceased-donor waiting list if their graft failed after an exchange transplant. Sönmez and Ünver 2015) propose the use of this incentivization procedure as follows: Since the waitlist is managed by UNOS, if the UNOS National Exchange Program is the only program, which can give compatible pairs the insurance, then they would only join the national program. Then most incompatible pairs would also join the UNOS National Program as well, as the best chance for them is where the compatible pairs participate. So if plausible conditions are satisfied, the UNOS program would emerge as the largest exchange program, whose patient volume is higher than all remaining programs combined.

International exchange programs are also being proposed, and successful early attempts are being made. For example, APKD has conducted an international kidney chain. Another intriguing proposal is using in exchange a compatible pair from a developing country that is 'de facto' incompatible, as the patient could not afford the transplant and its aftercare. Moreover, she could not even afford the on-going dialysis treatment. By paying for this patient's transplant and aftercare, Rees et al. (2017) reports saving 11 American patients through a kidney-exchange chain. Although clearly there will be ethical issues that need to be discussed related to this type of exchange, this proposal falls broadly in the cross-section of both creating larger pools for exchange and incentivizing compatible pairs to participate, which we discussed before.

One criticism against very large pools is whether there is a need to create the largest pool possible. It should be expected that there will be constant returns from scale exceeding certain pool sizes. Indeed, the marginal gains taper off and settle to a constant level in simulations in the limit if the pool is generated according to the underlying governing distributions. However, kidney exchange is a dynamic problem in its nature as pool sizes grow. Depending on the composition of the remaining patients in the pool after each exchange run, gains from large pools may never taper off due to past selection bias. Some of the papers we discuss on dynamic kidney exchange below deal exactly with this problem. 


\subsubsection{Dynamic Matching in Kidney Exchange}

Although most studies model it as a static problem, kidney exchange is a dynamic matching problem. Patient-donor pairs arrive over time, and some leave before being matched. Thus, using optimal algorithms designed for a static problem could sacrifice number of transplants that could potentially be conducted in a dynamic world. To measure such effects, several papers have been written.

Ünver (2010) shows that under large-market assumptions, the waiting costs can be minimized on certain occasions if all matches found are not conducted immediately, and sometimes some types of pairs should be held in order to match future incoming pairs. In particular, these types are A patient - B donor or B patient - A donor. However, opportunity costs of not doing dynamic optimization but making greedy matching seem to be small if the market is at some sort of steady state.

Anderson et al. (2015a) study a similar problem from a different perspective. They use random graph techniques where tissue-type probability is modeled as a random event and the pool consists mainly of hard-to-match patients. They find that when a new pair joins the pool, conducting a twoway or three-way exchange or continuing to an on-going altruistic-donor chain immediately with myopic foresight has a very low efficiency cost with respect to far-sighted dynamic optimization. However, matching for only two-way exchanges this way may create a problem.

Many exchange programs, on the other hand, run their computer algorithms for matching after a number of pairs accumulate, but not greedily. This may be due to administrative costs of running exchange or based on a heuristic that waiting for agents to accumulate is good. Akbarpour, Li, and Gharan (2013) consider a model to rationalize this type of behavior. Suppose patients expire and their expiration time is private information to the patients. They propose a matching mechanism that justifies waiting for the market to thicken while eliciting this private information as an approximately dominant strategy for the patients.

In the computer science literature, adaptive dynamic kidney exchange models that use nonparametric regression techniques on past data to determine optimal policy for the future have also been introduced. An important forerunner of this approach is Dickerson, Procaccia, and Sandholm (2012). There are several papers following this approach. These approaches are the easiest to implement in real life with the rising computer power, if indeed it can be shown that the gains from this approach are substantial over myopic static approaches. Until now, most of the empirical and theoretical evidence is that most of the gains can be exploited by using simple dynamic rules of thumb, such as not ending a donor chain with an AB donor, so that it is easy to find a future match for this donor.

Another dynamic approach is using time contracts. For example, can we help a patient now in return for a future benefit for a different patient? Sönmez, Ünver, and Yenmez (2017a)'s idea, discussed above, is such a futures contract. Indeed, Veale et al. (2017) reported 3 uses of a variant of such an intertemporal insurance scheme leading to 25 transplants through chain exchanges. This 
scheme is utilized as follows: The old living donor, paired with potential patient who will likely need a future kidney transplant, initiates a chain of exchanges now by donating to an incompatible pair. In return, the potential patient receives a guaranteed priority in the deceased-donor queue if her kidney indeed fails in the future. The donor has a short donation window due to her old age, and the insurance scheme helps other pairs to receive transplants through chain exchanges now, in addition to insuring the potential patient originally paired with the donor.

Also, we can think of a social-security scheme in which a pair donates today and receives an organ tomorrow. If the thickness of such a market is high enough, and trust to the system can be sustained, we can imagine participation could be high. A proposal in this direction is made in Ausubel and Morrill (2014).

\section{Liver Exchange}

The liver is another organ for which living donation is possible. It is the second-most transplanted organ following the kidney. Living donation requires more invasive surgery on donors than kidneys: A lobe of the liver is removed from the donor and transplanted to the patient. It is riskier than kidney donation. The larger the lobe taken from the donor as a ratio of the whole liver, the greater the risks for the donor. Once the initial transplant is successful and post-transplant problems are overcome, the graft in the patient and the remnant lobe in the donor both grow back in a short amount of time. Each part creates a healthy, full liver.

The liver consists of 8 anatomical parts: 4 of them make up what we will refer to as the 'left lobe' while the rest make up what we will refer to as the 'right lobe.' These two are the most commonly transplanted parts of the liver. Due to liver's asymmetric shape, the right lobe is on average at least $60 \%$ of its total volume. A patient needs a liver graft of at least $40 \%$ of her own liver size. Otherwise, she may die or have complications of 'too-small-for-size' syndrome. As a result, if the donor is smaller than the patient, in most cases right-lobe donation is the only feasible transplant.

The mortality risk to the donor is reported as 5 in 1000 transplants for right-lobe donations and 1 in 1000 for left-lobe transplants. There are also more morbidity risks associated with right-lobe donation (Lee, 2010).

Besides size compatibility, blood-type compatibility is the other medical compatibility requirement, as in the kidney. On the other hand, a liver transplant seems not to be adversely affected by possible tissue-type antibodies, unlike the kidney. So tissue-type incompatibility is not a problem.

Unlike kidney failure, there is no alternative treatment for liver failure (a kidney patient can go through dialysis in theory for a long time, although this is considered an inferior treatment method).

This made right-lobe donation a common practice in the medical community. However, a highprofile death of a right-lobe donor in 2003 in the US adversely affected the whole attitude toward living donation for all organs in the US. 
Since then, living donation for livers are mostly practiced in far eastern countries and Muslim countries where deceased donation is not common due to mostly cultural reasons. Per capita, South Korea, Japan, and Turkey seem to be leaders in living-donor liver donations (Lee et al., 2001). Moreover, hepatitis-caused liver failure seems to be quite common in these countries, making liver failure an important health concern.

Although tissue-type compatibility is not a concern, liver donation encounters an important road block because of size compatibility requirements in addition to blood-type compatibility. As a result, liver exchange has started in South Korea (as in the case of kidney exchange, they have beenpioneers in this modality) (Hwang et al., 2010). Besides South Korea, Hong Kong and Taiwan also have liver-exchange programs. Such transplants have been done also in Turkey.

There are two important contributions from liver exchange.

1. More patients can be saved due to liver-exchange transplants

2. Fewer right-lobe transplants may be necessary.

The first benefit is similar to kidney exchange, while the second one is more related to specifics

of liver exchange. As Sönmez, Ünver, and Yenmez (2017b) shows, these two objectives need not contradict each other.

Moreover, Ergin, Sönmez, and Ünver (2017b) show that an incentive-compatible mechanism can be designed to screen the donors/pairs who are willing to donate their right lobe and those who are not willing to take this risk, keeping Pareto efficiency of the outcome intact.

Another interesting proposal is combining the donor pools of kidney exchange and liver exchange so that a donor attached to a liver or kidney patient can donate either his liver lobe or kidney depending on what kind of an exchange is assembled. Even in large markets, gains could occur from these economies of scale. Hence a two-way exchange can include a liver pair and a kidney pair (not only a liver pair - liver pair or a kidney pair-kidney pair matches). The liver patient's donor donates her kidney to the kidney patient, and, in return, the liver patient receives a lobe of liver fromldd the kidney patient's donor. This was proposed by Dickerson and Sandholm (2014). This was also the first paper on liver exchange outside of the medical community.

\section{Dual-Donor Organ Exchange}

In theory, living-donor organ exchange can be used for any organ for which living donation is possible. There are a number of transplantation procedures that require two donors for each successful transplant. The number of patients who use these procedures is not at all small. As in the case of living donor liver transplantation, these techniques are mostly practiced in far-eastern countries or Muslim countries. Although the medical community has documented considerable numbers of living donations using these modalities, they have not explored the possibility of utilizing living-donor 

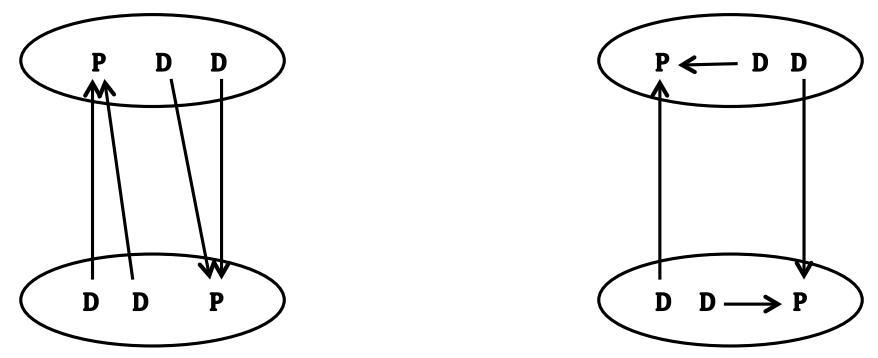

Figure 9: There are two types of possible two-way dual-donor exchanges

exchanges (with the exception of a single exchange).

There could be various combinations of exchange instead of just trading over a cycle as in the case of single-donor exchange. For example, there are two possible two-way exchange configurations (see Figure 9), while there are five possible three-way exchange configurations. In a two-way exchange, either patient may swap both of his donors or swap one of his donors, while the other donates directly to him. Hence, gains, which may not be possible through single-donor exchange, can be possible through dual-donor exchange. As two living donors are needed for each transplant, it is difficult for a patient to recruit two compatible donors. Therefore, gains from dual-donor organ exchange could be considerably greater than kidney exchange and maybe liver exchange.

These modalities were proposed by Ergin, Sönmez, and Ünver (2017a). The three main applications of dual-donor organ exchange are as follows:

1. Dual-graft liver exchange,

2. lung exchange, and

3. simultaneous liver-kidney exchange

We will talk about each of them below:

\subsection{Dual-Graft Liver Exchange}

Although in most cases a single donor is needed for liver transplant, because of the size compatibility requirement many times a patient has multiple donors who are blood-type compatible but each of them by herself cannot donate to the patient. Let's explain further what we mean by this. Two cases may occur:

1. Each donor is really small in size, and hence, even if either of them donated her left lobe alone, it would not be sufficient in volume for the patient.

2. One of the donors is as large as the patient, or his right lobe could be sufficient in size for transplantation. However, the volume of the left lobe of the donor falls below $30 \%$ of total 
liver volume. This threshold is considered the lowest acceptable remnant liver volume for the donor. Hence, the left lobe is too small, the right lobe is a good size, but it is simply not acceptable to harvest her right lobe from the donor due to risks.

South Korea has the highest nnumber of liver transplants per capita worldwide, with 942 livingdonor transplants (and approximately 50 million in population) in 2015. South Koreans introduced dual-graft liver transplantation in 2000, conducting 176 of these procedures in the period from 2011 to 2015, and they introduced single-graft liver exchange in 2003. As such, all key factors are exceptionally favorable in South Korea for a possible market-design application of dual-graft liver exchange. Indeed, one such conducted exchange was recently reported (see Jung et al., 2014). As an added bonus, most dual-graft liver transplants in South Korea are carried out at a single hospital, namely the ASAN Medical Center in Seoul. Performing by far the largest number of liver transplants worldwide, with more than 4000 liver transplantations to date, ASAn's success rate for one-year survival of patients receiving a liver transplant is $96 \%$ compared to the US average of $88 \%$

(Jung and Kim, 2015). Simulations in Ergin, Sönmez, and Ünver (2017a) suggest that an organized dual-graft liver exchange could increase the number of living-donor liver transplants by as much as $30 \%$ through two-way and three-way exchanges. This increase corresponds to saving as many as 100 patients annually if exchange is restricted to the ASAN Medical Center alone, and to saving as many as 300 patients annually if exchange can be organized throughout South Korea.

\subsection{Lung Exchange}

Just as South Koreans lead in innovations in living-donor liver transplantation, Japanese lead in living-donor lung transplantation. Sato et al. (2014) report that there is no significant difference in patient survival between living-donor and deceased-donor lung transplantations. In living-donor lobar lung transplantation, two donors each donate the lower lobe of one of their lungs. These two lobes are placed in the two lung cavities of the patient, then enlarge (though they do not grow new tissue) and function as lungs with reduced oxygen capacity. The lobes should be large enough to be transplanted into the cavities of the patient. Thus, size compatibility is an important constraint.

With the exception of occasional cases at Keck Hospital of the University of Southern California, the vast majority of living-donor lung transplantations are performed in Japan. Living-donor transplantation in general is more widely accepted in Japan than deceased-donor transplantation, although donations by deceased donors have significantly increased since the revised Japanese Organ Transplant Law took effect in July 2010 (Sato et al., 2014). For the case of lung transplantation, there have been more transplants from deceased donors in recent years than from living donors. The revision of the organ transplant law, the invasiveness of the procedure, and the high rate of incompatibility among willing donors all contribute to this outcome. Despite these factors, 20 of 61 lung transplants in Japan were from living donors in 2013 (Sato et al., 2014). Living-donor lung transplants to date have been mostly concentrated at two hospitals, with nearly half performed at Okayama University Hospital and another third at Kyoto University Hospital. 
While the potential for establishing an organized lung exchange is less clear than for an organized dual-graft liver exchange, Okayama University Hospital could be an idealized place for such an exchange program. That is because they conducted the most number of living-donor transplants in the world and have surpassed the global five-year survival rate lung transplant recipients of $50 \%$ with $82 \%$ for their patients $(87 \%$ for recipients from living donors) 8 One of the challenges faced in Japan has been the lack of precedence for living-donor organ exchanges. While kidney exchange is not illegal in Japan, it has so far not been culturally accepted. Instead, the members of the Japanese kidney transplantation community have been focusing on alternative strategies to utilize gifts of living donors through techniques such as blood-type incompatible kidney transplantation via desensitization medications .9 For the case of lung exchange, however, the gains from exchange could be huge in Japan in part due to the difficulty of finding two compatible donors and in part due to a lack of similar strategies for lung transplantation.

\subsection{Simultaneous Liver-Kidney Exchange}

Many liver patients suffer also from kidney failure. Nadim et al. (2012) note that simultaneous liver-kidney (SLK) transplants are the best option for many such patients. In the US, about $10 \%$ of all liver transplants are SLK transplants.

Two natural candidates for an organized SLK exchange are South Korea and Turkey. These two countries have some of the highest living-donation rates worldwide for both livers and kidneys. Based on the most recent data available from the International Registry for Organ Donation and Transplantation, South Korea was the worldwide leader in living-donor liver transplants per capita in 2013, followed by Turkey. The same year, Turkey was the worldwide leader in living-donor kidney transplants per capita, whereas South Korea had the fifth highest rate ${ }^{10}$ Living-donor SLK transplants, kidney exchanges, and single-graft liver exchanges are all performed in both countries. Hence, all three factors for an organized SLK exchange are favorable in both countries. An even more efficient, but also more ambitious, possibility would be a joint kidney-and-liver-exchange program that organizes

1. kidney exchanges,

2. liver exchanges, and

3. simultaneous liver-kidney exchanges.

\footnotetext{
${ }^{8}$ Information obtained from "100 Lung Transplants," Okayama University e-bulletin, Volume 2, January 2013, http://www.okayama-u.ac.jp/user/kouhou/ebulletin/pdf/vol2/news_001.pdf.

${ }^{9}$ Desensitization using medications is very expensive and time consuming. Recall the papers we discussed, Chun, Heo, and Hong (2016) and Andersson and Kratz (2016), that incorporate blood-type incompatible transplantation into kidney exchange.

${ }^{10}$ Data available at http://www.irodat.org/img/database/grafics/newsletter/IRODaT\%20Newsletter\% $202013 \% 20 . p d f$.
} 
This more elaborate program would allow an SLK patient to exchange a liver donor with a liver patient and/or a kidney donor with a kidney patient, potentially resulting in a higher number of transplants than three separate exchange programs in aggregate. Simulations in Ergin, Sönmez, and Ünver (2017a) suggest that an organized SLK exchange has the potential to quadruple the number of living-donor SLK transplants through two-way and three-way exchanges in this especially favorable scenario.

\section{Conclusion}

Since ethical constraints have prevented the sale of human organs in most countries, medical doctors, ethicists, and economists have come up with pseudo-markets that resemble barter-exchange marketplaces to mitigate the organ shortage problem. This can be viewed as a second-best solution within the realm of the ethical constraints. Computer scientists followed suit to engineer good, fast algorithms to facilitate such solutions whenever needed. Unlike many other problems, organ allocation is a dynamic and continuous problem, which requires constant attention from economists as new ethical and medical possibilities become available over time. Thus, it is difficult to write a definitive survey of such a fast-changing landscape. Here we tried to give our perspective of market design for organ exchanges as of mid-2017.

Once upon a time, microeconomics had promoted the establishment of competitive markets with monetary payments as a remedy for almost every resource-allocation problem without even discussing market-specific details except commonly known market failures, such as existence of excess market power and externalities, or the need for public goods provision and taxation. Even after mechanism design and game theory matured as tools of microeconomics, they almost always answered quite abstract questions. Therefore, microeconomics used to be more of a descriptive branch of economics rather than a prescriptive one for most of scarce resource-allocation problems. This sometimes lead policymakers to think of economics as a field out of touch with the realities of life and the human condition. Clearly, for suitable environments the market perspective should be kept in mind as one of the most important insights of all of economics (as we know by the second fundamental theorem of welfare economics). However, it looks like those were past days. Livingdonor kidney exchanges have turned into a success story of market design, a field of microeconomics. And it continues to produce many policy proposals that can and should be implemented to save lives of kidney, liver, and lung patients all over the world.

Besides organ exchange, many barter-exchange websites are popping up on the internet for various forms of economic activity despite the lack of any barriers to prevent monetary transactions. Our knowledge and experience in organ exchanges would also be useful for such marketplaces.

Moreover, there are on-going efforts to institute markets with possible monetary compensation for both deceased and living-human organs. Someday these may succeed. Even then, we think that the tools and experiences we have learnt in designing and implementing living-donor organ 
exchanges will continue to be valuable. Still, there will be patients who will not be able to participate in market transactions. Many tools we have discussed can be tweaked to be used in conjunction with a monetary market.

While discussing the ins and outs of legal human organ sales, the important theoretical and empirical questions are what would be the projected welfare improvement through a regulated market with respect to currently available modalities; who would benefit and who would lose from such a transition; and whether the predicted welfare improvements would be enough to justify crossing over important ethical barriers. Without answering these questions satisfactorily, it may be difficult to take sides in this important debate.

\section{References}

Abdulkadiroğlu, Atila and Tayfun Sönmez (1999). "House allocation with existing tenants." Journal of Economic Theory, 88, 233-260.

Abraham, David, Avrim Blum, and Tuomas Sandholm (2007). "Clearing algorithms for barter exchange markets: enabling nationwide kidney exchanges." Proceedings of ACM-EC 200\%: the Eighth ACM Conference on Electronic Commerce.

Akbarpour, Mohammad, Shengwu Li, and Shayan Oveis Gharan (2013). "Thickness and information in dynamic matching markets." Working paper.

Anderson, Ross, Itai Ashlagi, David Gamarnik, and Yash Kanoria (2015a). "Efficient dynamic barter exchange." Working paper.

Anderson, Ross, Itai Ashlagi, David Gamarnik, et al. (2015b). "Kidney exchange and the alliance for paired donation: operations research changes the way kidneys are transplanted." Interfaces, 45 (1), 26-42.

Andersson, Tommy and Jörgen Kratz (2016). "Kidney exchange over the blood group barrier." Working paper.

Ashlagi, Itai and Alvin E. Roth (2014). "Free riding and participation in large scale, multi-hospital kidney exchange." Theoretical Economics, 9, 817-865.

Ashlagi, Itai, David Gamarnik, Michael A. Rees, and Alvin E. Roth (2012). "The need for (long) chains in kidney exchange." Working paper.

Ashlagi, Itai, Felix Fischer, Ian A. Kash, and Ariel D. Procaccia (2015). "Mix and match: a strategyproof mechanism for multi-hospital kidney exchange." Games and Economic Behavior, 91, $284-296$.

Ausubel, Lawrence M. and Thayer Morrill (2014). "Sequential kidney exchange." American Economic Journal: Microeconomics, 6 (3), 265-85.

Becker, Gary S. and Julio Jorge Elías (2007). "Introducing incentives in the market for live and cadaveric organ donations." Journal of Economic Perspectives, 21 (3), 3-24.

Cantillon, Estelle (2017). "Broadening the market design approach to school choice." Oxford Review of Economic Policy, forthcoming. 
Caragiannis, Ioannis, Aris Filos-Ratsikas, and Ariel D. Procaccia (2015). "An improved 2-agent kidney exchange mechanism." Theoretical Computer Science, 589, 53-60.

Chun, Youngsub, Eun Jeong Heo, and Sunghoon Hong (2016). "Kidney exchange with immunosuppressants." Working paper.

De Klerk, Marry, Karin M. Keizer, Frans H. J. Claas, et al. (2005). "The Dutch national living donor kidney exchange program." American Journal of Transplantation, 5 (9), 2302-2305.

Dickerson, John P, Ariel D Procaccia, and Tuomas Sandholm (2012). "Dynamic matching via weighted myopia with application to kidney exchange." $A A A I$.

Dickerson, John P. and Tuomas Sandholm (2014). "Multi-organ exchange : the whole is greater than the sum of its parts." Proceedings of the Association for the Advancement of Artificial Intelligence.

Dickerson, John P. and Tuomas Sandholm (2016). "Organ exchanges: a success story of AI in healthcare." Thirtieth Conference on Artificial Intelligence Tutorial Forum.

Dougherty, C J (1987). "Body futures: the case against marketing human organs." eng. Health Prog, 68 (5). KIE: 13 fn.; KIE: KIE BoB Subject Heading: organ and tissue donation; KIE: Full author name: Dougherty, Charles J, 51-55.

Edmonds, Jack (1965). "Paths, trees, and flowers." Canadian Journal of Mathematics, 17, 449-467.

Ergin, Haluk, Tayfun Sönmez, and M. Utku Ünver (2017a). "Dual-donor organ exchange." Econometrica, forthcoming.

Ergin, Haluk, Tayfun Sönmez, and M. Utku Ünver (2017b). "Efficient and incentive compatible liver exchange." Working paper in progress.

Ferrari, Paolo, Linda Cantwell, Joseph Ta, et al. (2017). "Providing better-matched donors for HLA mismatched compatible pairs through kidney paired donation." Transplantation, 101 (3).

Gallai, Tibor (1963). "Kritische Graphen II." Magyar Tud. Akad. Mat. Kutato Int. Kozl., 8, 373395.

Gallai, Tibor (1964). "Maximale Systeme unabhangiger kanten." Magyar Tud. Akad. Mat. Kutato Int. Kozl., 9, 401-413.

Gentry, S. E., D. L. Segev, M. Simmerling, and R. A. Montgomery (2007). "Expanding kidney paired donation through voluntary participation by compatible donors." American Journal of Transportation, 7 (10), 2361-2370.

Goyal, M, RL Mehta, LJ Schneiderman, and AR Sehgal (2002). "Economic and health consequences of selling a kidney in india." JAMA, 288 (13), 1589-1593.

Huh, Kyu Ha, Myoung Soo Kim, Man Ki Ju, et al. (2008). "Exchange living-donor kidney transplantation: merits and limitations." Transplantation, 86 (3).

Hwang, Shin, Sung-Gyu Lee, Deok-Bog Moon, et al. (2010). "Exchange living donor liver transplantation to overcome ABO incompatibility in adult patients." Liver Transplantation, 16 (4), $482-490$.

Jevons, William S. (1876). Money and the Mechanism of Exchange. D. Appleton and Co., New York. 
Jung, Dong-Hwan, Shin Hwang, Chul-Soo Ahn, et al. (2014). "Section 16. update on experience in paired-exchange donors in living donor liver transplantation for adult patients at asan medical center." Transplantation, 97.

Jung, Min-Ho and Eil-Chul Kim (2015). "A doctor who transplants life." The Korea Times, October 11, URL http://www.koreatimes.co.kr/www/news/nation/2016/03/668_188409.html.

Kerstein, Samuel J. (2009). "Autonomy, moral constraints, and markets in kidneys." The Journal of Medicine and Philosophy: A Forum for Bioethics and Philosophy of Medicine, 34 (6), 573.

Kranenburg, Leonieke W., Willij Zuidema, Willem Weimar, et al. (2006). "One donor, two transplants: willingness to participate in altruistically unbalanced exchange donation." Transplant International, 19 (12), 995-999.

Lee, Sung-Gyu (2010). "Living-donor liver transplantation in adults." British Medical Bulletin, 94 (1), 33-48.

Lee, Sung-Gyu, Shin Hwang, Kwang-Min Park, et al. (2001). "An adult-to-adult living donor liver transplant using dual left lobe grafts." Surgery, 129 (5), 647-650.

Li, Shengwu (2017). "Ethics and market design." Oxford Review of Economic Policy, forthcoming. Manlove, David F. and Gregg O'Malley (2012). "Paired and altruistic kidney donation in the UK: algorithms and experimentation." Experimental Algorithms: 11th International Symposium, SEA 2012, Bordeaux, France, June 7-9, 2012. Proceedings. Ed. by Ralf Klasing. Berlin, Heidelberg: Springer Berlin Heidelberg, pp. 271-282.

Melcher, M. L., J. P. Roberts, A. B. Leichtman, A. E. Roth, and M. A. Rees (2016). "Utilization of deceased donor kidneys to initiate living donor chains." American Journal of Transplantation, 16 (5), 1367-1370.

Montgomery, R. A., S. E. Gentry, W. H. Marks, et al. (2006). "Domino paired kidney donation: a strategy to make best use of live non-directed donation." Lancet, 368, 419-421.

Nadim, M. K., R. S. Sung, C. L. Davis, et al. (2012). "Simultaneous liver-kidney transplantation summit: current state and future directions." American Journal of Transplantation, 12 (11), 2901-2908.

Nicolò, Antonio and Carmelo Rodriguez-Álvarez (2017). "Age-based preferences: incorporating compatible pairs into paired kidney exchange." Games and Economic Behavior, forthcoming.

Okumura, Yasunori (2014). "Priority matchings revisited." Games and Economic Behavior, 88, 242-249.

Rapaport, F. T. (1986). "The case for a living emotionally related international kidney donor exchange registry." Transplantation Proceedings, 18 (3), 5-9.

Ratner, Lloyd E., Abbas Rana, Emily R. Ratner, et al. (2010). "The altruistic unbalanced paired kidney exchange: proof of concept and survey of potential donor and recipient attitudes." Transplantation, 89, 15-22.

Rees, M. A., T. B. Dunn, C. S. Kuhr, et al. (2017). "Kidney exchange to overcome financial barriers to kidney transplantation." American Journal of Transplantation, 17 (3), 782-790.

Rees, Michael A., Jonathan E. Kopke, Ronald P. Pelletier, et al. (2009). "A non-simultaneous extended altruistic donor chain." New England Journal of Medicine, 360, 1096-1101. 
Ross, L. F. and E. S. Woodle (2000). "Ethical issues in increasing living kidney donations by expanding kidney paired exchange programs." 69, 1539-1543.

Ross, L. F., D. T. Rubin, M. Siegler, et al. (1997). "Ethics of a paired-kidney-exchange program." New England Journal of Medicine, 336, 1752-1755.

Roth, A. E., T. Sönmez, M. U. Ünver, F. L. Delmonico, and S. L. Saidman (2006). "Utilizing list exchange and non-directed donation through 'chain' paired kidney donations." American Journal of Transplantation, 6 (11), 2694-2705.

Roth, Alvin E. (2007). "Repugnance as a constraint on markets." Journal of Economic Perspectives, $21(3), 37-58$.

Roth, Alvin E., Tayfun Sönmez, and M. Utku Ünver (2004). "Kidney exchange." Quarterly Journal of Economics, 119 (2), 457-488.

Roth, Alvin E., Tayfun Sönmez, and M. Utku Ünver (2005a). "A kidney exchange clearinghouse in New England." American Economic Review Papers and Proceedings, 95, 376-380.

Roth, Alvin E., Tayfun Sönmez, and M. Utku Ünver (2005b). "Pairwise kidney exchange." Journal of Economic Theory, 125 (2), 151-188.

Roth, Alvin E., Tayfun Sönmez, and M. Utku Ünver (2005c). "Transplant center incentives in kidney exchange." Harvard University and Koç University, unpublished mimeo.

Roth, Alvin E., Tayfun Sönmez, and M. Utku Ünver (2007). "Efficient kidney exchange: Coincidence of wants in markets with compatibility-based preferences." American Economic Review, 97 (3), 828-851.

Sack, Kevin (2012). "60 lives, 30 kidneys, all linked." New York Times, February 18, 2012; http://www.nytimes.com/2012/02/19/health/lives-forever-linked-through-kidneytransplant-chain-124.html.

Saidman, Susan L., Alvin E. Roth, Tayfun Sönmez, M. Utku Ünver, and Francis L. Delmonico (2006). "Increasing the opportunity of live kidney donation by matching for two- and three-way exchanges." Transplantation, 81 (5), 773-782.

Sato, Masaaki, Yoshinori Okada, Takahiro Oto, et al. (2014). "Registry of the Japanese Society of Lung and Heart-Lung Transplantation: Official Japanese lung transplantation report, 2014." General Thoracic and Cardiovascular Surgery, 62 (10), 594-601.

Scheper-Hughes, Nancy (2005). "The ultimate commodity." The Lancet, 366 (9494), 1349-1350.

Segev, Dorry, Sommer Gentry, Daniel S. Warren, Brigette Reeb, and Robert A. Montgomery (2005). "Kidney paired donation: optimizing the use of live donor organs." Journal of the American Medical Association, 293, 1883-1890.

Shapley, Lloyd and Herbert Scarf (1974). "On cores and indivisibility." Journal of Mathematical Economics, 1, 23-37.

Sönmez, Tayfun and M. Utku Ünver (2011). "Matching, allocation, and exchange of discrete resources." Social Economics. Ed. by Jess Benhabib, Alberto Bisin, and Matthew O. Jackson. Vol. 1A. Handbooks in Economics. Elsevier, pp. 781-852.

Sönmez, Tayfun and M. Utku Ünver (2013). "Market design for kidney exchange." The Handbook of Market Design. Ed. by Zvika Neeman Alvin E. Roth and Nir Vulkan. OUP, pp. 93-137. 
Sönmez, Tayfun and M. Utku Ünver (2014). "Altruistically unbalanced kidney exchange." Journal of Economic Theory, 152, 105-129.

Sönmez, Tayfun and M. Utku Ünver (2015). "Enhancing the efficiency of and equity in transplant organ allocation via incentivized exchange." Available as Boston College Working Paper 868.

Sönmez, Tayfun, M. Utku Ünver, and M. Bumin Yenmez (2017a). "Incentivized kidney exchange." Working paper.

Sönmez, Tayfun, M. Utku Ünver, and M. Bumin Yenmez (2017b). "Transaction frictions in organ exchange." Working paper in progress.

Sönmez, Tayfun, M. Utku Ünver, and Özgür Yılmaz (2016). "How (not) to integrate blood subtyping technology to kidney exchange." Working paper.

Steinberg, David (2011). "Compatible-incompatible live donor kidney exchanges." Transplantation, 91 (3).

Taylor, James Stacey (2005). Stakes and Kidneys: Why Markets in Human Body Parts are Morally Imperative. Live Questions in Ethics and Moral Philosophy. Routledge.

The Authors for the Live Organ Donor Consensus Group (2000). "Consensus statement on the live organ donor." Journal of the American Medical Association, 284 (22), 2919-2926.

Toulis, Panos and David C. Parkes (2015). "Design and analysis of multi-hospital kidney exchange mechanisms using random graphs." Games and Economic Behavior, 91, 360 -382.

Ünver, M. Utku (2010). "Dynamic kidney exchange." Review of Economic Studies, 77 (1), 372-414.

Veale, Jeffrey L., Alexander M. Capron, Nima Nassiri, et al. (2017). "Vouchers for future kidney transplants to overcome 'chronological incompatibility' between living donors and recipients." Transplantation, Online First.

Veatch, R. M. (2006). "Organ exchanges: fairness to the O-blood group." American Journal of Transplantation, 6 (1), 1-2.

Zargooshi, J (2001). "Quality of life of Iranian kidney 'donors'." The Journal of Urology, 166 (5), 1790-1799. 